\title{
Nanoparticles for brain-specific drug and genetic material delivery, imaging and diagnosis
}

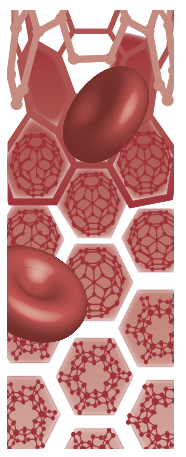

The poor access of therapeutic drugs and genetic material into the central nervous system due to the presence of the blood-brain barrier often limits the development of effective noninvasive treatments and diagnoses of neurological disorders. Moreover, the delivery of genetic material into neuronal cells remains a challenge because of the intrinsic difficulty in transfecting this cell type. Nanotechnology has arisen as a promising tool to provide solutions for this problem. This review will cover the different approaches that have been developed to deliver drugs and genetic material efficiently to the central nervous system as well as the main nanomaterials used to image the central nervous system and diagnose its disorders.

First draft submitted: 18 September 2015; Accepted for publication: 26 January 2016; Published online: 16 March 2016

Keywords: blood-brain barrier $\bullet$ brain $\bullet$ drug delivery $\bullet$ imaging $\bullet$ nanoparticles $\bullet$ siRNA

CNS diseases represent the largest and fastest growing area of unmet medical needs. Over one and a half billion people worldwide suffer from CNS disorders. Despite all efforts, however, treatments and adequate diagnoses for CNS diseases remain limited due to the inability of most therapeutic and contrasting agents to adequately cross the blood-brain barrier (BBB). This makes it more important than ever to find ways to deliver therapeutic drugs and genetic material to the CNS effectively, safely and conveniently. In this regard, nanoparticles have emerged as the major alternative to conventional methods since they can be used to maintain drug levels in the therapeutic range, and to increase the half-life, solubility, stability and permeability of drugs [1,2]. The utility of nanoparticles has also boosted the development of new imaging and diagnostic agents for assessing brain function and diagnosing CNS disorders and diseases through the use of structural imaging techniques such as MRI, functional MRI, computed axial tomography, positron emission tomography, magnetoencephalography and optical imaging. The nanoparticles are multifunctional molecules and can be used in drug and genetic material delivery as well to deliver imaging agents and diagnostic molecules (Figure 1).

This review summarizes the characteristics of the BBB and its crossing by different nanoparticles and considers the most relevant uses of nanoparticles for brain-specific drug and genetic material delivery as well as for diagnostic procedures. The last few years have seen the publication of very useful reviews on the use of nanoparticles for diagnostic and therapeutic purposes in the CNS [3-5].

\section{The blood-brain barrier}

The $\mathrm{BBB}$ provides a natural defense against circulating toxic or infectious substances, mediates efflux of waste products and maintains the ionic and fluid balance protecting the brain from fluctuations in ionic composition [6].

\section{BBB structure}

The BBB is a specialized substructure of the vascular system composed of the capillary
Inmaculada Posadas ${ }^{1,2}$, Silvia Monteagudo ${ }^{1,2}$ \& Valentín Ceña*,1,2

'Unidad Asociada Neurodeath, Facultad de Medicina, Universidad de Castilla-La Mancha, Albacete, Spain

${ }^{2}$ CIBERNED, Instituto de Salud Carlos III, Madrid, Spain

*Author for correspondence:

Tel.: +34 680222322

valentin.cena@gmail.com
Future fsg 


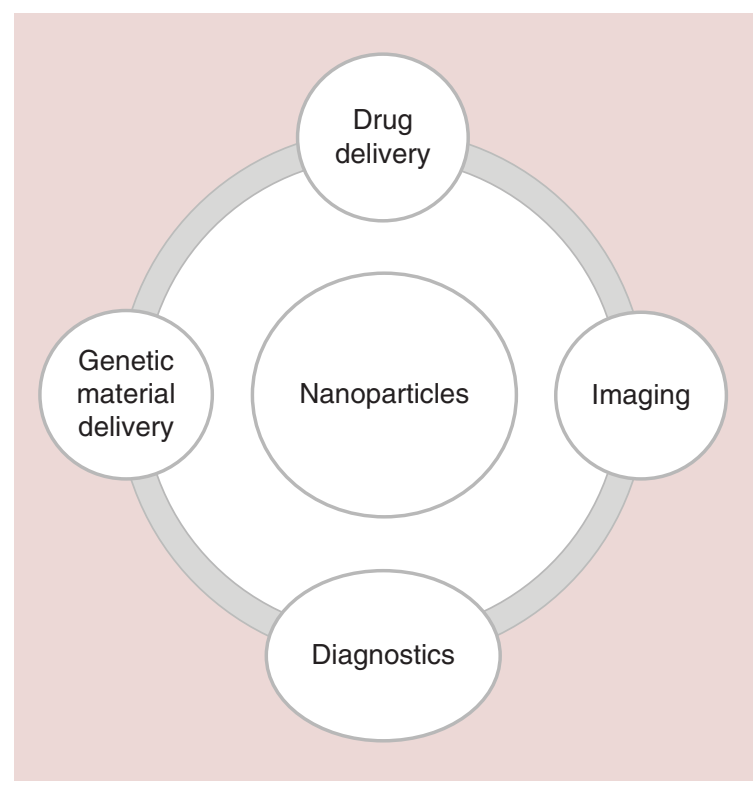

Figure 1. Scheme showing the multifunctionality of the nanoparticles that can have different biomedical applications such as drug delivery, diagnostics, genetic material delivery or imaging.

endothelial cells that line the cerebral microvessels and the surrounding perivascular elements, including the basal lamina, pericytes, astrocyte endfeet and interneurons. The most characteristic feature of the $\mathrm{BBB}$ is the presence of tight junctions between adjacent endothelial cells, leading to a very high transendothelial electrical resistance (about $8000 \Omega \times \mathrm{cm}^{-2}$ ). The $\mathrm{BBB}$ also represents a metabolic barrier since it contains enzymes that are capable of metabolizing and inactivating different drugs and toxic and neuroactive compounds [6,7].

\section{Mechanisms involved in BBB crossing}

The structure of the BBB only allows the passive diffusion of some gases, water and hydrophobic molecules with a molecular weight (MW) of less than 400-500 $\mathrm{Da}$. Thus, most of the nutrients and endogenous components including essential fatty acids, amino acids, glucose or vitamins are transported into the brain by different saturable transporters expressed at the BBB [7].

The majority of nanosized systems that can cross the BBB use adsorptive-mediated transcytosis and receptor-mediated transcytosis as the two major mechanisms for nanoparticle delivery across the BBB. The most common strategy used to facilitate the transport of nanoparticles into the CNS has consisted of incorporating a targeting molecule or binding it to blood molecules that are recognized by receptors present at the brain endothelium, mediating endocytosis or transcytosis (Figure 2). Several endogenous BBB receptors have been considered for CNS-targeted drug delivery, such as receptors for transferrin (Tf), insulin, glutathione, low-density lipoprotein (LDL) and LDL receptorrelated protein-1 and -2 (LRP-1, LRP-2) and angiopep-2 peptide $[6,8,9]$. Drugs conjugated with ligands for these receptors, such as peptides or peptidomimetic monoclonal antibodies (mAbs), have been widely used to target the delivery of nanoparticles. After transcytosis, the nanoparticles diffuse into the brain extracellular space before being endocytosed by parenchyma cells in the brain. However, the nanoparticles can be also removed from the brain by the P-glycoprotein (P-gp) efflux system and by the high cerebrospinal fluid turnover rate.

\section{Nanoparticles}

Current strategies used for drug delivery to the brain include invasive delivery, temporary disruption of the BBB as well as the use of drug-delivery systems. While direct injection in selected cases can be an effective invasive modality for local delivery (e.g., in some tumors), it is not efficient for brain metastasis or neurodegenerative diseases, which require therapeutic agents to be spread throughout the brain. Reversible opening of the BBB by an osmotic or a chemical method allows therapeutic agents to enter the brain; however, this approach can also result in significant damage to the brain. In comparison, selective delivery of diagnostic and therapeutic molecules to the whole brain through the vascular route using drug-delivery systems is much less invasive and it is potentially safe. Nanoparticles can be used to maintain drug levels in a therapeutically desirable range, as well as to increase the half-lives, solubility, stability and permeability of drugs.

The use of nanoparticles has boosted the development of new imaging and diagnostic agents for assessing brain function and diagnosing CNS disorders. Nanoparticles in brain imaging can be divided into two major categories: nanosized imaging agents and nanocarriers functionalized with imaging agents such as gadolinium. They are also capable of carrying other functional entities if necessary, leading to theranostic molecules. The following nanoparticles have been used to deliver therapeutic or diagnostic molecules to the CNS (Figure 3):

\section{Natural polymers}

Polymers are macromolecules having large chains containing a variety of functional groups and that can be conjugated with other low- and high-MW materials. Natural polymers have been used for the controlled release, enhanced stability and improved bioavailability of drugs and genetic material. The main characteristic of these nanoparticles for drug delivery is that 


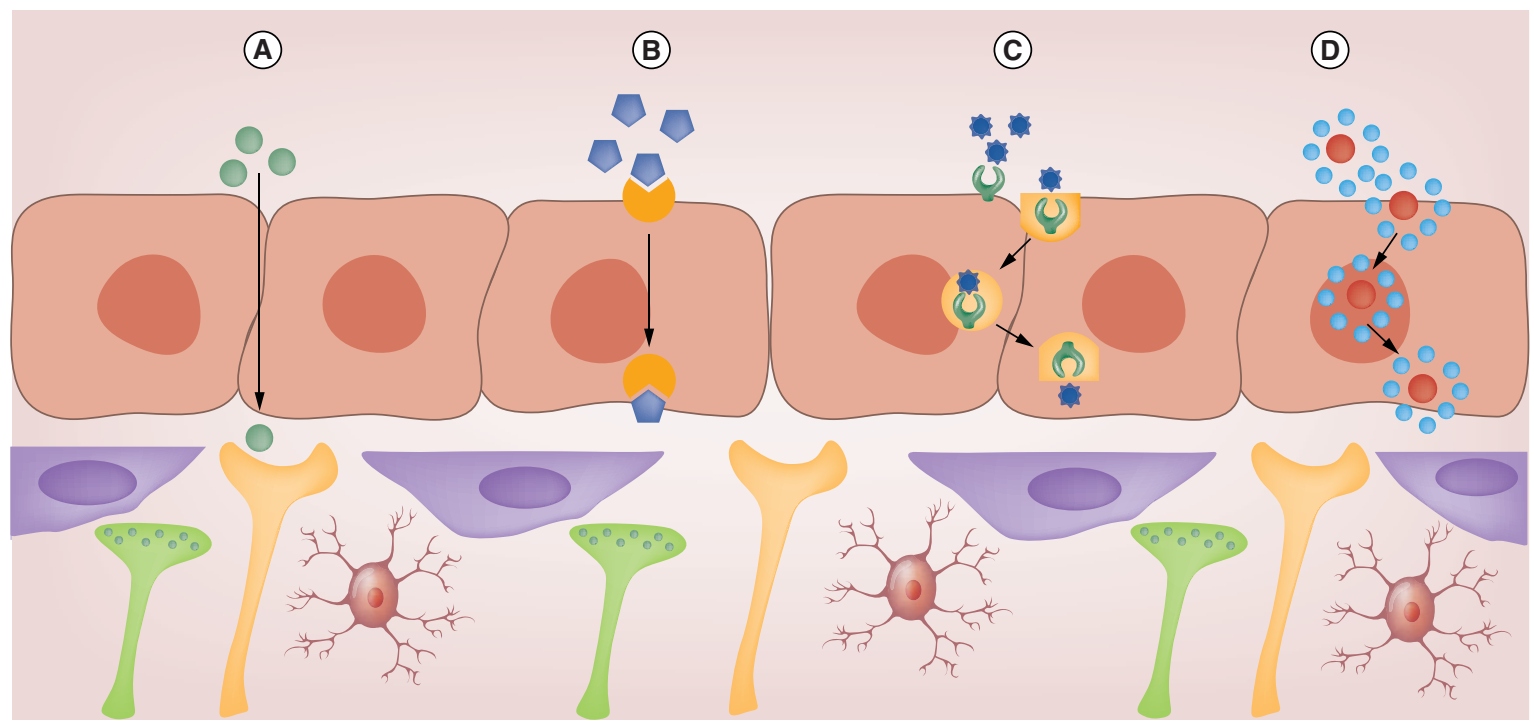

Figure 2. The different mechanisms that can be used by the nanoparticles to cross the blood-brain barrier.

(A) Transcellular lipophilic pathway. (B) Transport proteins. (C) Receptor-mediated transcytosis and (D) adsorptive transcytosis.

hydrophobic compounds can be physically encapsulated into micellar nanoparticles and be protected from degradation and blood clearance after in vivo administration.

Chitosan is a naturally occurring, biodegradable, biocompatible polysaccharide that has the ability to efficiently form nanoparticles. Chitosan nanoparticles can be used to deliver peptides (amyloid-beta subfragments), dopamine and caspase inhibitors to the CNS following systemic administration. Furthermore, chitosan particles can be surface modified to display a variety of ligands for BBB bypass, including transferrin receptor antibodies.

\section{Drug \& genetic material delivery to the CNS}

Chitosan nanoparticles easily enter into neuronal cells, predominantly by endocytosis and accumulate around the cell nucleus. However, their low water solubility often leads to decreased transfection efficiency $[9,10]$. To overcome this problem, chitosan nanoparticles have been linked to different molecules to increase their solubility. For example, complexation of chitosan with PEI resulted in lower toxicity and higher transfection efficiency than chitosan alone in culture cells $[9,11]$. Similarly, different biocompatible chitosan-based films and microspheres made of chitosan have been designed for growth factor delivery to the CNS and to serve as a structural support for transplanted cells to promote regeneration of the CNS after injury [9,12]. In vivo, Tween 80-coated chitosan nanoparticles improved gallic acid brain targeting and the tolerance profile of the drug as compared with the free drug when administered intraperitoneally and orally to rats [13].
Collagen-based nanoparticles have been also used as gene, drug and cell-delivery systems because of their low antigenicity and their ability to protect the cargo from the body environment. In situ gelling type I collagen hydrogel has been used to deliver glial-derived growth factor to the CNS. The collagen hydrogel allowed glial-derived growth factor secretion into the striatal parenchyma, after stereotatic injection into the rat striatum and significantly reduced the host brain's response at the delivery site.

\section{Lipids \& cationic lipids}

Liposomes are vesicles composed of one (unilamellar) or several (multilamellar) lipid bilayers surrounding internal aqueous compartments. Relatively large amount of drug molecules can be incorporated into the aqueous compartment (water-soluble compounds) or lipid bilayers (lipophilic compounds). Conventional liposomes are rapidly removed from circulation by the reticuloendothelial system (RES), which leads to fast systemic elimination. The circulation time can be extended by decreasing the particle size $(<100 \mathrm{~nm})$ and by liposome-surface modification with polyethylene glycol (PEG) [14]. Pegylation allows these liposomes, named Stealth ${ }^{\circledR}$ liposomes, to escape from mononuclear phagocyte system and to extend blood-circulation time [15]. However, a smaller size also increases the risk of potential pulmonary toxicity [16].

\section{Drug \& genetic material delivery to the CNS}

Liposomes are probably the most studied and clinically recognized drug nanocarriers owing to their long track record, low toxicity and ability to deliver both 


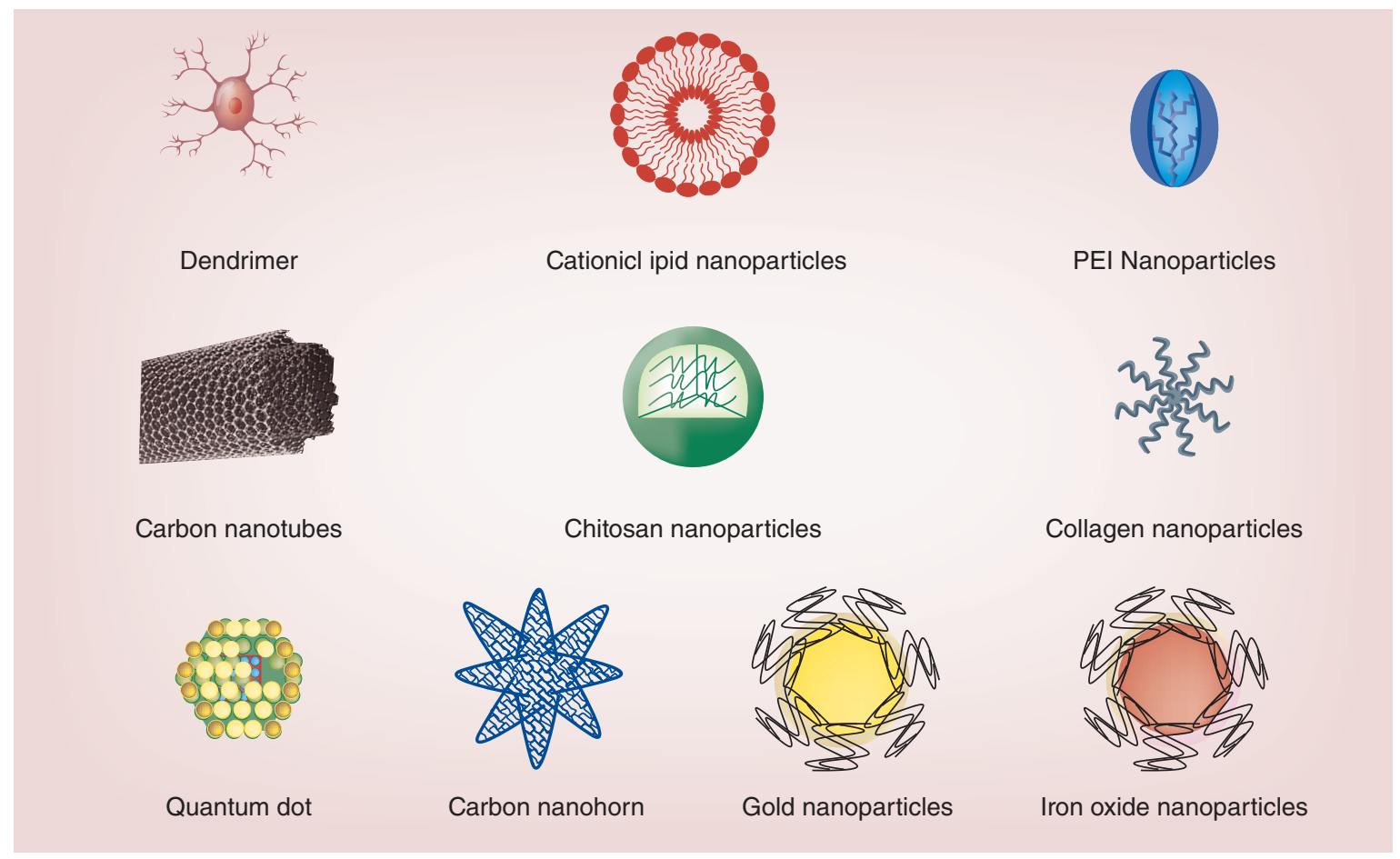

Figure 3. The different types of nanoparticles reviewed in this article.

hydrophilic and lipophilic compounds reasonably well. Consequently, research has been conducted into the use of liposomal formulations to deliver various drugs into the CNS, including chemotherapeutic compounds, antiretrovirals [17], anti-ischemia drugs and drugs used to treat Alzheimer disease. Moreover, liposomes have been combined with ultrasound-mediated $\mathrm{BBB}$ disruption to increase the antineoplastic efficacy of several liposomal formulations in brain tumors after intravenous administration [17].

The potential of liposomes for CNS delivery is related to the ease of surface modification for targeted delivery. For instance, to target the brain selectively, liposomes can be additionally modified (vectorized) with mAbs to create immunoliposomes. OX26 mAbinmunoliposomes favor receptor-mediated transcytosis across the $\mathrm{BBB}$ in vivo without altering $\mathrm{BBB}$ integrity [17]. Similarly, liposomes conjugated with anti-EGFR antibodies were used to deliver sodium borocaptate into EGFR-overexpressing glioma cells in vitro and in brain tumor bearing mice. In this case, intravenous administered immunoliposomes penetrated in solid tumor, where the BBB is disrupted, but not in normal tissues of CNS indicating that EGFRconjugated immunoliposomes could not pass through intact BBB [18]. For the same purpose, liposomes have been functionalized with peptides, or with transferrin. However, transferrin transporters are also present in liver and bone marrow, limiting the selectivity and application of these compounds [18].
Solid lipid nanoparticles generally consist of a matrix of lipids that remain solid at room and body temperature. These solid lipids are mostly physiological lipids such as fatty acids, mono-, di- or triglycerides, glycerine mixtures and waxes and require surfactants for stabilization. These nanocarriers facilitate the sustained, controlled release of therapeutic agents and have a low toxicity. Solid lipid nanoparticles have been widely used as drug delivery nanocarriers for the CNS, mainly in brain tumors, but also in CNS infected with HIV and in AD models [19,20].

Cholesterol self-assembled nanoparticles have been also used to deliver drugs to the CNS. Cholesterolbased solid nanoparticles combined with esterquat and stearylamine increased the BBB permeability of saquinavir, estimated with an in vitro model of a monolayer of human brain-microvascular endothelial cells, which could result in a more efficient drug delivery into the brain [21].

Cationic lipids, which are commonly comprised of a polar headgroup and nonpolar symmetric or asymmetric carbon-based tail, condense and protect nucleic acids from degradation in the extracellular environment. They can bind and compact DNA or RNA by electrostatic interactions between the positively charged lipid assemblies and the negatively charged nucleic acids, generating cationic lipid-DNA/RNA complexes referred to as lipoplexes. Once the target tissue is reached after systemic administration, the lipoplexes are endocytosed, the lipid component 
of the lipoplex fuses with the endosomal vesicle and quickly releases the nucleic acid into the cytoplasm. However, lipid nanoparticles display a low efficiency of small interference RNA (siRNA) delivery due to a reduced escape from the endocytic vesicles [22]. The in vivo performance of a lipidic nanocarrier depends on the administration route, meaning different administration routes must be optimized to achieve relevant vector and cargo levels at the target tissue. Several studies have reported that the intravenous administration of cationic liposome/DNA or cationic liposome/ cationic lipid/DNA produces systemic gene expression, particularly in the lungs, but not in the brain. An additional problem that appears after the intravenous administration of cationic lipids is vector aggregation in the presence of serum, leading to vector disintegration, genetic material release and degradation. These issues, together with the low efficiency of expression mediated by cationic lipids compared with viral vectors, have led to the need for local injections in order to obtain significant transfection in the CNS.

One of the possible solutions for the genetic material delivery obstacle is 'Trojan Horse Liposome' (THL) technology [23]. This technology uses the transcytosis pathway to enter the brain avoiding BBB disruption. THL is comprised of immunoliposomes carrying genetic material, engineered with known lipids containing PEG, which stabilizes its structure in vivo. The tissue target specificity of THL is given by conjugating approximately $1 \%$ of the PEG residues to peptidomimetic MAbs that bind to specific endogenous receptors for transferrin or insulin located on both the BBB and the brain cellular membranes, respectively. Applications of THLs include tissue-specific gene expression with tissue-specific promoters, and brain expression of therapeutic genes in animal models of Parkinson's disease [24]. Moreover, liposomes have been coupled to a peptide that acts as a neuronal addressing molecule by binding to acetylcholine receptors to deliver siRNA to the CNS when administered intravenously [25].

Other approaches recently described to increase liposome-mediated transfection efficiency include the use of a new series of pyridinium cationic lipids, with a dopamine backbone attached via an ether linkage to the hydrophobic alkyl tails, that efficiently and selectively transfect dopaminergic neurons from the nucleus accumbens, and cortical neurons from the frontal cortex, in primary coculture of neurons and glial cells [26].

\section{Synthetic polymers}

Synthetic polymeric nanoparticles are colloidal systems made of polymers with a compact structure, where the therapeutic agent is either entrapped within the colloid matrix or coated on the particle surface by conjugation or adsorption. Polyethyleneimine (PEI), poly-L-lysine (PLL), poly-acrylate, poly-(D,L-lactide) (PLA), poly(glycolic acid) (PGA) and poly(D,L-lactide-co-glycolide) (PLGA) are some of the synthethic polymers that have been used to CNS delivery successfully. PEI is an organic polymer with a high cationic-charge density potential due to the presence of multiple amino groups within its backbone that can be protonated and therefore, can bind nucleic acids electrostatically. They exist in two forms: linear or branched. PEI will spontaneously adhere and condense nucleic acids to form toroidal complexes that are readily endocytosed by cells. In addition, PEI is able to retain an important buffering capacity at virtually any cellular $\mathrm{pH}$, protecting nucleic acids and avoiding lysosomal nuclease degradation. However, one of the major problems described for PEIs is that they can be toxic to certain cell types, including neurons, depending on $\mathrm{MW}$, structure and concentration of the molecule used. In fact, high-MW PEIs cause necrotic-like changes due to alterations in the plasma membrane, followed by activation of a mitochondrial-mediated apoptosis in several cell types [27,28]. In contrast, lowMW PEIs displayed much less toxicity but almost no transfection efficiency. This suggests that PEI toxicity appears to be related to the disruption of the endosome/lysosome complex, the same mechanism responsible for their transfection effectivity since the buffer capacity of these protonable polymers cause excess proton accumulation in endosomes, which leads to counterion and water accumulation resulting in osmolysis. A great deal of effort has been devoted to improving the biocompatibility of high-MW PEIs by modifying their solubility, biodegradability and chemical homogeneity. PLL is a cationic polymer that contains positively charged hydrophilic amino group consisting in amino-acid lysine as a repeat unit. PLL polymers are biodegradable and rapidly released from endosomes, but show a low level of transfection efficiency and suffer from immunogenicity and toxicity caused by its amino-acid backbone [29]. Poly-acrilate polymers are polymers based on the structure of acrylic acid, which consists of a vinyl group and a carboxylic acid terminus. Poly(acrylic acid) and poly(methacrylicacid) are commonly used $\mathrm{pH}$-responsive polyacids. Among the different derivatives, Poly(butylcyanoacrilate) (PACA) nanoparticles have the ability to absorb or encapsulate a wide range of drugs and have succeeded in development of nanoparticles for intravenous administration [30]. However, the utility of PACA nanoparticles is limited because the biocompatibility and the release rate for drugs is still deficient. In addition, the most commonly used poly-acrylate polymers are not hydrolytically degradable and often are associated with 
neurotoxicity. PLA, PGA and PLGA have the advantages of biocompatibility and biodegradability while releasing active drug molecules to the site of action. PLGA, which results from random copolymerization of PLA and PGA, is the most investigated degradable polymer for biomedical applications and has been used in sutures, drug delivery devices and tissue engineering scaffolds depending on the composition of the PLGA. However, bulk erosion of the polymer limits the significant modulation of the release rate [31].

Polymeric nanoparticles, in the absence of surface modification with target molecules, have a limited capacity to cross the BBB. As a matter of fact, more work is still needed to achieve efficient polymersbased transfection vectors able to cross the BBB when administered intravenously.

\section{Drug \& genetic material delivery to the CNS}

PEI copolymers have been used to deliver drugs and genetic material to CNS cells in recent years. It has been established that PEIs with a branched structure condense nucleic acids to a greater extent than linear PEIs.

Direct intracranial brain injection of DNA/PEI complexes produces transgene expression levels comparable to those obtained with the lenti- or adenoviral vectors in the brains of newborn and adult mice and rats. Nevertheless, transfection efficiency is highly reduced when PEI vectors are administered intravenously. This is due to particle aggregation, unspecific interactions of these particles with blood components, such as plasma proteins, uptake by the RES or opsonization by the innate immune system. Moreover, an excess of positive charge can strongly activate the complement system. To increase transfection in vivo, the coupling of hydrophilic polymers (such as PEG) to DNA/PEI complexes has been investigated. Covalent coupling of PEG protects from particle aggregation, in keeping with data previously reported for liposomes. PEGylation also prevented undesirable effects reported for non-PEGylated complexes, including erythrocyte aggregation and binding of complexes to plasma proteins when injected intravenously [32]. Another strategy developed to increase complex solubility in vivo consisted of coupling PEIs with a cationic carrier, such as polyethylene oxide or Pluronic 123. However, intravenous administration of this compound in $\mathrm{C} 57 \mathrm{BI} / 6$ mice resulted in luciferase expression in the liver, heart, spleen and lung, whereas no activity was detected in the brain [33]. In addition, lumbar intrathecal injection of PEGylated PEI can deliver the gene to regions distant from the injection site [34], although this method is too traumatic to be used in human therapy.
The most extensively used polymeric nanoparticles for CNS drug delivery are those made of acrylic polymers that have been used in models of various CNS diseases, such as brain tumors, brain HIV infection and Alzheimer's. PACA nanoparticles offer the advantage of being rapidly degraded in vivo, which could reduce the toxicity caused by polymer accumulation in the CNS. PACA nanoparticles coated with polysorbate 80 overcome the BBB successfully without altering cell viability and BBB integrity [35]. Polysorbate 80 is thought to adsorb to apolipoprotein $\mathrm{B}$ and $\mathrm{E}$ on brain capillary epithelium and facilitate brain uptake via receptor-mediated endocytosis [36]. In addition, intraperitoneal as well as oral PACA nanoparticles administration can be use for brain targeting [37].

Polyester-based nanoparticles could offer some advantages over acrylic polymers: they could be potentially safer for brain drug delivery as the degradation products are mainly water and carbon dioxide. Nanoparticle systems made of polyesters have been extensively used for CNS drug delivery [38]. Several attempts have been made to use different cationic block copolymers as drug-delivery system to the CNS, such as PEI-poly(ethylene glycol) or PLL-PEG covalently cross-linked with superoxide dismutase 1 (SOD1). These complexes, when injected into mice, have shown to increase the enzyme-nanoparticle accumulation in brain tissues, although the potential to attenuate oxidative stress in neurodegenerative diseases has not been clearly established [39]. PLL-poly(ethylene glycol) administered by oropharyngeal aspiration mediate effective gene delivery to the brain, eyes and lung. Nevertheless, most of the nanoparticle administered is retained by sputum precluding the efficient delivery of cargo to the epithelium [40].

Similarly, cationic nanogels formed by PEI segments connected by disulfide bridges have been used to encapsulate nucleoside reverse transcriptase inhibitors. In vivo administration of nanogel-nucleoside reverse transcriptase inhibitors decorated with a peptide binding brain-specific apolipoprotein E receptor resulted in increased brain accumulation, and high retroviral activity in a humanized mouse model of HIV-1 infection in the brain [41]. In addition, PEI copolymers have been also used to transfect stem cells, an example of this being retinoic acid-PEI complex nanoparticles inducing embryonic stem cell-derived neuronal differentiation [42]. On the other hand, PLA, PLG and the copolymer PLGA, which seems to be advantageous due to its biocompability and biodegradability, have been reported to be appropriate for cargo delivery across the BBB. PLGA loperamide-loaded nanoparticles, functionalized with an $\mathrm{mAb}$ against the transferrin receptor, efficiently crossed the BBB and exerted 
analgesic effects when administered intravenously [43]. Similarly, intravenous administration of polysorbate 80(PS 80)-PLGA nanoparticles loaded with methotrexate-transferrin conjugates resulted in successful migration across the BBB with low organ toxicity and high antitumor activity [44].

\section{Imaging \& diagnosis}

PEI copolymers attached to poly(glycidyl methacrylate) containing superparamagnetic iron oxide $\left(\mathrm{Fe}_{3} \mathrm{O}_{4}\right)$ nanoparticles have been used as a magnetic resonance contrast (MRI) agent (Figure 4). However, the intramedullary or intravitreal administration of these nanoparticles showed that nanoparticles remained close to the injection site, and only a small amount entered the axons and were transported to neuronal somata [45].

PS 80-poly(methacrylic)acid nanoparticles have been used for the delivery of BBB-impermeable imaging agents such as gadolimium. MRI showed that Gdnanoparticles were able to cross the BBB and enter the brain in healthy mice. Further, suggest that PS 80 might play a role in nanoparticle transport across the $\mathrm{BBB}$ to gain entry into the CNS [46].

\section{Dendrimers}

Dendrimers are repeatedly branched polymer molecules containing a cascade of branches grown from one or several cores. Dendrimers grown from a central core adding repeated branching cycles, each of which represents a new 'generation' of dendrimer, being usually classified by generation number (i.e., generation 4 refers that the branching reactions are performed onto the core molecule four-times). Dendrimers contain three architectural domains: the core, to which the branches are attached; the shell of the branches surrounding the core; and the multivalent surface formed by the branches' termini [47]. This characteristic structure confers to dendrimers interesting properties including easily functionalization, well-defined reactive groups on the particle surface, versatility in the composition and structurally controlled nanoscale building [48], which has made them very promising nanomaterials for diagnostic and therapeutic purposes.

To date, more than 200 kinds of dendrimers and dendrons have been synthesized that can be grouped into different families, including the poly(amidoamine) (PAMAM), poly(propyleneimine) (PPI), poly(triazene), polyamidoether, phosphodendrimers, poly(thiophosphate), a dendritic family based on the 2,2-bismethylolpropionic acid (bis-MPA) monomer and poly(biphenylpropyl) families. A comprehensive description of the dendrimer families was recently published [49].
Dendrimers possess the ability of transporting molecules across cell membranes or biological barriers by being endocytosed via a clathrin- and caveolaemediated cellular internalization. Once inside the cell, dendrimers act as a proton sponge due to the presence of secondary and tertiary amines on their surface. They escape from endosomes and lysosomes, releasing their cargo to the cytosol [50]. Depending on the charge of the functional groups present at the surface, dendrimers can be divided mainly into polycationic dendrimers, able to form polyplexes with nucleic acids at physiological $\mathrm{pH}$ and polyanionic dendrimers, able to bind cationic drugs or to coordinate into platinum complexes [51].

PAMAM dendrimers are the most studied type of dendrimer for biological applications, including drug and genetic material delivery to the CNS. The systemic administration of PAMAM dendrimers has shown minimal brain uptake in healthy animals. PAMAM dendrimers below generation 6 are rapidly cleared from systemic circulation by kidney filtration or by the RES, whereas PEGylated dendrimers or PAMAM dendrimers above generation 6 tend to circulate longer and be distributed to peripheral organs $[52,53]$. Only when BBB permeability is increased, as happens during neuroinflammation or meningitis, is the access of these dendrimers to the CNS favored. However, in the absence of meningeal inflammation, CNS penetration of PAMAM dendrimers is scarce and even after intraventricular or subarachnoid administration, most of them are retained and line the ventricles within the ependymal layer [48]. Interestingly, dendrimer-Nacetylcysteine (NAC) conjugates may enable improved drug delivery and achieve the concentration of NAC in the brain required for neuroprotection. The administration of a hydroxil-functionalized G4 PAMAMNAC dendrimer improved motor function and attenuated neurological injury in a model of hypothermic circulatory arrest [53].

Improving the $\mathrm{BBB}$ permeability of dendrimers has been addressed by coupling specific BBB-targeting ligands onto the surface of dendrimers. As was mentioned above, receptor-mediated endocytosis is a natural pathway through which endogenous proteins traverse the BBB. Transferrin, EGF, rabies virus glycoprotein (RVG29) and angiopep2 peptides are some of the ligands that have been used to design CNS drug-delivery systems. Among these, angiopep2 peptides have been reported to possess the highest brain transcytosis capacity and parenchymal accumulation. Generation 5 PAMAM dendrimers labeled with NIR fluorophore and multiple copies of angiopep2 peptides were developed to study the regulation of $\mathrm{BBB}$ permeability. Intravenous administration of these nanopar- 


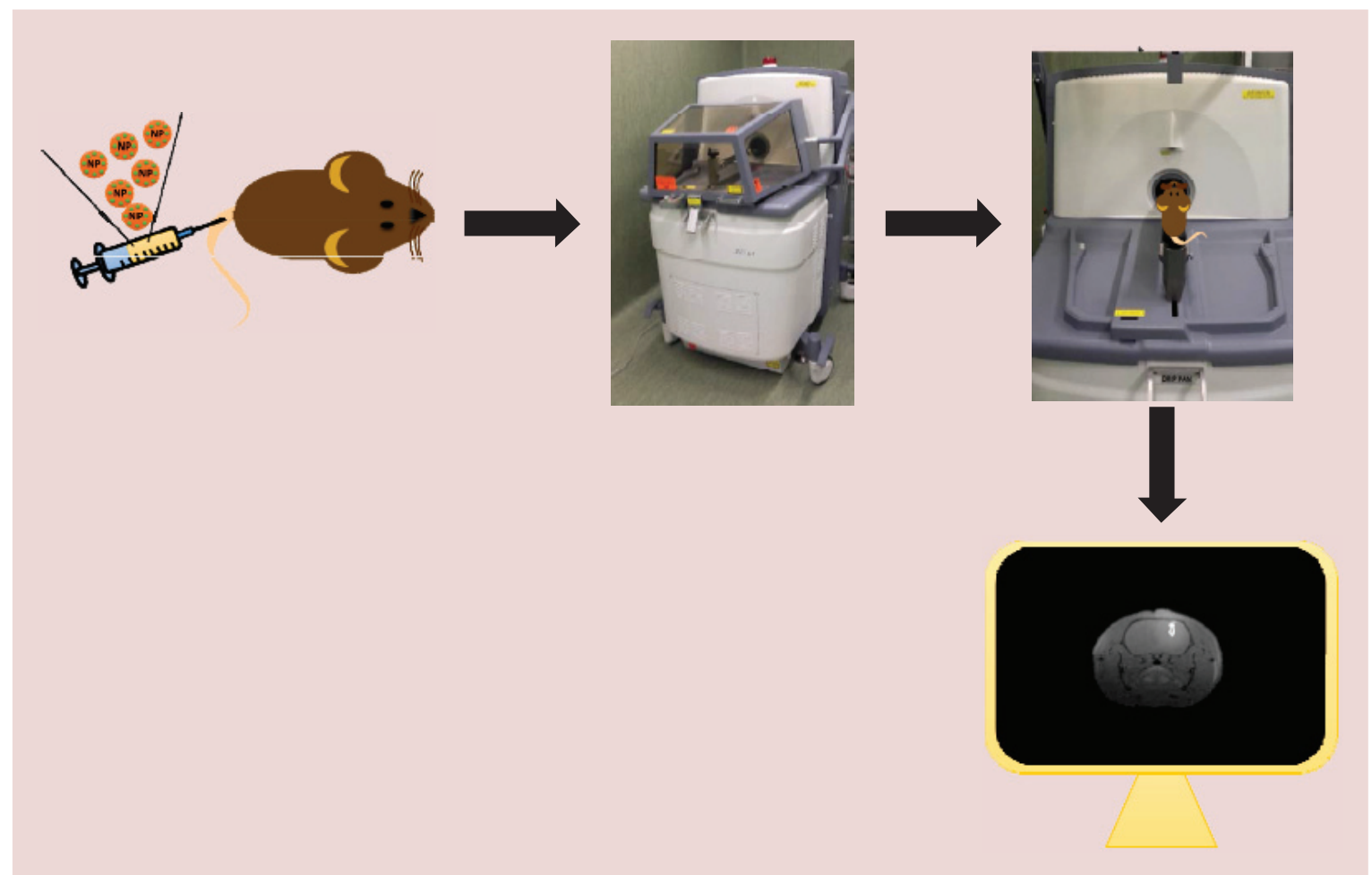

Figure 4. Identification of the location in the brain of the injected nanoparticle. The nanoparticle decorated with an NMR probe is injected in the tail vein of the mouse and the animal placed in an NMR machine. The detector returns the image with the precise location of the labeled nanoparticle.

ticles led to higher concentrations in the liver, spleen and kidney. In addition, all nanoparticles labeled with angiopep2 peptides demonstrated higher cerebral uptakes than the control nanoparticles, with the concentrations being higher in the hippocampus, striatum and cerebellum than in the cerebral cortex [54].

Dendrimers are one of the most extensively investigated nanoparticles for a wide range of biomedical applications including brain drug delivery, imaging and diagnosis. However, a serious drawback of dendrimers is their toxicity, mainly related to the positive charge present on the surface of the cationic dendrimers. PAMAM dendrimers exhibit neuronal toxicity. For example, unmodified PAMAM G5- $\mathrm{NH}_{2}$ induces mitochondrial membrane potential depolarization in neurons and astroglial cells [55] and activates autophagy [56]. PAMAM dendrimers also increase reactive oxygen species and DNA damage in a generation-dependent manner [57]. Similar toxicity has been also reported for unmodified PPI dendrimers. In addition, the nondegradability of PAMAM/PPI-type dendrimers is a major concern for their biological use. However, appropriate modifications of the surface of the dendrimers can greatly reduce their toxicity, with the transformation of PAMAM amine groups into pyrrolidone derivatives resulting in minor toxic activity in vitro [58]. Moreover, surface modification of den- drimers leads to a more efficient in vivo administration. Hydroxyl-functionalized generation-4 PAMAM dendrimers selectively localized in neurons and activated microglia in the injured brain and retina when administered intravenously to newborn rabbits [59], or to 6-12-month-old dogs [53]. One of the problems that arise following the functionalization of dendrimers to reduce toxicity is the observed decrease in diffusion and in cellular uptake of the dendrimers and their payload. The functionalization of PAMAMs (G4G12 PAMAM) dramatically reduces diffusion in the CNS and dendrimer penetration in living neurons [48]. Moreover, the PEGylation of dendrimers results in improved biocompatibility, reduced immunogenicity, increased water solubility, prolonged half-life and increased protection against nuclease, thus protecting siRNA cargo from degradation [60].

\section{Drug \& genetic material delivery to the CNS}

Although dendrimers have shown an excellent profile as gene carriers in vitro in several cell lines and primary cortical neurons, astrocytes, microglia and oligodendrocytes showing low toxicity and suitable transfection efficiency [61], the use of dendrimers for CNS drug delivery is currently under research mainly because BBB crossing continues to pose a problem for the in vivo use of dendrimers. 
Up to date, major studies using dendrimers as drugdelivery systems in CNS disease in vivo models are related to brain tumors where the BBB is disrupted. Most of the effort has been devoted to the development of dendrimers that can specifically target the brain tissue, such as by synthesizing thiamine (Tm)-conjugated PPI dendrimers for increased delivery of paclitaxel (PTX) across the BBB. Biodistribution studies of these compounds showed a higher biodistribution of TmPPI conjugates into the brain and in vivo pharmacokinetics studies revealed significant slow clearance of PTX from the body [62].

However, modifications of the dendrimer molecules are necessary to facilitate $\mathrm{BBB}$ crossing in the absence of a disease-induced increase in permeability. To achieve this task, proteins, peptides and other specific target molecules have been coupled to dendrimers. Peptides targeting the low-density lipoprotein receptor-related protein-1 (LRP1), such as angiopep-2, possess high brain penetration capability and exhibit high transcytosis capacity and parenchymal accumulation. They have been used to decorate dendrimers to increase their $\mathrm{BBB}$ crossing ability, leading to a high expression of the target gene in the brain and the spleen [63].

Apart from PAMAM dendrimers, other dendrimers such as PPI and carbosilane have been investigated for biomedical applications. Poly-lysine dendrimers (G3 and G5) were found in the periventricular zone and in the cytoplasm of neurons and glial cells in the forebrain and hippocampus after intraventricular administration to rats [64]. More recently, it has been also reported that a second-generation carbosilane dendrimer transported siRNA efficiently into the brain, crossing the BBB [65].

A different approach consists of incorporating lower generation dendrimers onto elongated magnetic nanoparticles (dendriworms) to deliver siRNA to the CNS [66]. Intracranial infusion using an osmotic pump demonstrated that dendriworms were able to penetrate through the brain parenchyma, but unfortunately, intravenous administration of dendriworms in mice preferentially accumulated in the lungs and reticuloendothelial filtration organs, with low amounts reaching the brain [66].

Besides chemical modification of dendrimers to allow brain delivery of their cargo after intravenous injection, other possible routes of administration are being explored. Intranasal drug administration allows direct delivery of drugs to the brain. This administration route was recently used to deliver HMGB1 siRNA using a biodegradable PAMAM dendrimer, producing a significant depletion of the target gene in many brain regions, including the prefrontal cortex and striatum. More importantly, it markedly decreased infarct volume in the postichemic rat brain [67].
Imaging \& diagnosis

The development of dendritic nanoparticle-mediated brain imaging and diagnosis has attracted considerable attention. PAMAM dendrimer generations 1-8 have been functionalized with gadolinium and rhodamine $\mathrm{B}$ for both magnetic resonance and fluorescence imaging of malignant gliomas. Several more attempts have been made to generate dendrimer-based imaging nanoprobes, including a dendrimer-PEG copolymer-based architecture for fluorescence sensing, imaging for the first time extracellular $\mathrm{pH}$ in the brain in a mouse epilepsy model [68]; a dendrimer-based optical/paramagnetic nanoprobe, which showed a capability to circumvent the $\mathrm{BBB}$ and yielded high sensitivity images of brain tumors in vivo [69] and the use of dendritic phosphorescent probes for oxygen imaging in rat brains [70].

The clinical diagnosis of brain tumors using MRI is a very sensitive diagnostic method that is highly dependent on contrast agents. Currently, the widely used low MW contrast agents such as gadolinium-diethylene triamine pentaacetic acid (DTPA) suffer from rapid renal clearance, nonspecificity, and low contrast efficiency. A macromolecular MRI-contrast agent based on dendrigraft poly-L-lysines uses chlorotoxin as a tumor-specific ligand and gadolinium-diethylene triamine pentaacetic acid as the payload. The MRI images showed that the signal enhancement was maintained much longer than using standard contrast agents, which might allow for a more exact diagnosis of tumors in animal models of xenografted brain [71]. More recently, two-order targeted nanoprobes containing MRI/optical imaging reporters, tumor vasculature targeted cyclic (RGDyK) peptides and BBB-permeable Angiopep-2 peptides coupled to a PAMAM-G5 dendrimer were able to efficiently cross the intact BBB in normal mice. They also precisely delineated the boundary of an orthotopic U87MG human glioblastoma brain xenograft with a high target-to-background signal ratio. Overall, this two-order targeted nanoprobe might provide the possibility for real-time optical-image-guided brain tumor resection during surgery [72].

\section{Carbon-based nanoparticles}

Fullerene, carbon nanotubes (CNTs) and carbon nanohorns $(\mathrm{CNHs})$ [73] are the main materials representing carbon nanotechnology. CNTs are graphite sheets rolled into single or multiwalled tubes with a diameter between $0.5-20 \mathrm{~nm}$ and a length of $1.5-5000 \mathrm{~nm}$ [74]. Due to their inherent properties, these nanomaterials are playing an increasing role in different fields of nanomedicine, including the delivery of drugs, genes, vaccines and also in thermotherapy of tumors due to their thermal conductivity [75]. In spite of the prominence of carbon-based materials in the nanotechnol- 
ogy literature, exploration of their application to CNS therapeutics is at a very early stage.

The use of carbon-based nanomaterials has, at the present moment, two main disadvantages: lack of solubility and potential toxicity. As produced, CNTs are insoluble in most organic or aqueous solvents; therefore, for any type of biological application the nanotube's surface needs to be functionalized. Indeed, this first drawback is currently being solved by incorporating different pendant units that increase solubility, using covalent and noncovalent functionalization of the CNT surface. However, the toxicity of CNTs remains an issue in biomedical applications. These materials can induce lipid peroxidation and oxidative stress, as well as pulmonary toxicity [76]. Regarding brain toxicity, it has been proposed that microglia content determines brain-specific toxicity of CNTs. Furthermore, they are hydrophobic and tend to aggregate in bodily fluids. Before CNTs are used to treat brain disorders, issues such as their CNS penetration and toxicity need to be addressed.

\section{Drug \& genetic material delivery to the CNS}

Carbon-based nanoparticles (CNHs) have been used in vivo to deliver drugs and genetic material to peripherally xenografted tumors with varying results. While the intravenous administration of $\mathrm{CNH}$ s to mice with subcutaneously transplanted tumors resulted in $\mathrm{CNHs}$ accumulation in macrophages and endothelial cells, and only a few $\mathrm{CNH}$ s were found in the tumors [73], oxidized single-walled CNTs combined with vincristine and wrapped in DSP-PEG-IGF-IR mAbs efficiently enhanced drug delivery to a peripheral xenografted tumor and also reduced the toxicity of vincristine to the living mice [77]. However, although intravenous administration of $\mathrm{CNH}$ s led to peripheral distribution accumulating in the liver, spleen, blood, tumor and skin, $\mathrm{CNH}$ s were not detected in brain [73] indicating that unmodified CNHs do not cross BBB. In agreement with this, Kafa et al. have recently described that multiwalled carbon nanotubes (MWNTs) are able to cross BBB in vitro models, but intravenous administration of MWNTs only resulted in $1 \%$ accumulation in brain 5 min after postinjection [79].

Stereotaxic administration of amino-functionalized carbon nanotubes $\left(\mathrm{CNT}_{-} \mathrm{NH}_{3}+\right)$ into mouse brain cortex resulted in early internalization within the microglia, although severe nanotube structure deformation leading to partial degradation of CNTs also occurred [80]. Moreover, CNT-mediated siRNA knock down of caspase 3 ameliorated the motor deficits observed in a rodent model of stroke [81]. Furthermore, the stereotaxic administration of two different aminofunctionalized MWNT showed rapid incorporation into different neuronal cells including microglia, astrocytes and neurons. However, the administration of both constructs led to a local induction of inflammatory cytokines at the injection site [82].

CNTs offer many potential advantages over other types of nanoparticles used or developed for cancer therapy. For example, covalently functionalized CNTs are able to evade the endosomal compartment and translocate directly into the cytoplasm of different types of cells [83]. Their large surface area and internal volume also allows drugs and a variety of small molecules, such as contrast agents, to be loaded onto the nanotube. However, the use of CNTs for CNS drug delivery is currently at an early stage. Progress toward preclinical and clinical trials will depend on the outcomes of efficacy and toxicology studies, which will provide the necessary risk-to-benefit assessments for carbon-nanotube-based materials.

\section{Quantum dots}

Quantum dots (QDs) are semiconductor nanoscale crystalline structures formed by combining chemical elements from groups II, III and V of the periodic table. Structurally, QDs are colloidal sheets, composed of a metalloid crystalline core and a shell that shields the core, rolled into single or multiwall tubes with sizes ranging from 2 to $10 \mathrm{~nm} \mathrm{[6].} \mathrm{QDs} \mathrm{exhibit} \mathrm{several} \mathrm{advanta-}$ geous optical properties, such as long-term photostability and bright fluorescence over narrow and size-tunable emission bands, allowing for multiplexing with multiple emission wavelengths from a single excitation source [84]. The emission bandwidth is very narrow, while the excitation bands are broad, allowing for excitation with a wide variety of laser and light sources. QDs are generally hydrophobic and therefore require a wide variety of surface functionalization with PEG, lipids and dextrans in order to render them soluble in biologically relevant solvents. Moreover, the surface of QD can be decorated with different functional groups like carboxylic acids, primary amines and thiols, which can be used for conjugation with biomolecules such as antibodies, nucleic acids or peptides by either covalent or noncovalent interactions [6]. To date, the most common core/shell used in biological and medical research includes $\mathrm{CdSe} / \mathrm{ZnS}$, $\mathrm{CdSe} / \mathrm{ZnCdS}, \mathrm{CdTe} / \mathrm{CdSe}$ and $\mathrm{InP} / \mathrm{ZnS}$, although their toxicity, including oxidative stress and cytotoxicity, likely limited their application in vivo [78].

\section{Drug \& genetic material delivery to the CNS}

QDs have been used for drug and gene delivery to the CNS based on their ability to cross the BBB. In this direction, the combination of QDs with transferrin and the highly active antiretroviral therapy drugs saquinavir or amprenavir, increases the solubility and bioavailability of the drugs, resulting in good crossing 
of the BBB and significant inhibition of HIV-1 in the brain [6]. QDs can be also used to transfect neurons. It has been reported that PEGlyated quantum rods (QRs) with different surface functional groups (amine and maleimide) allowed the delivery of siRNAs to human neuronal cells, and led to drastic knockdown of specific gene expression [85].

Several attempts have been made to selectively target QDs to a specific cell type, including streptavidincoated QDs that selectively target the nanoparticle to microglia [86]. Endosomal escape is a key factor for nanoparticle-induced efficient transfection and conjugation of QDs, with some peptides, like those derived from the parent peptide JB577, not only mediating cytosolic delivery in brain tissue but also facilitating efficient endosomal escape [87]. Similarly, conjugation of QDs with PEG-appended dyhydrolipoic acid (DHLA) and linked to the peptide Palm1 allowed the QDs to be taken up by different culture cells and readily escape the endosome to the soma [88].

\section{Imaging \& diagnosis}

QDs have emerged as a revolutionary imaging technology and have found diverse biomedical applications, including some in the CNS. Over the last few years there have been many examples of QDs used to determine brain tumor boundaries using both passive and targeted delivery methods. QDs may be linked to antibodies, peptides and nucleic acids for their use as specific-target fluorescence probes in vivo. QDs can be used to selectively label tumors by conjugating them to antibodies raised against specific proteins located in the tumoral cells. To increase brain delivery of QDs, the nanoparticles have been incorporated into the bilayer membrane of liposomes. Although QDs provide very high contrast and tumor localization, the main drawback to their use is that the long-term effects of QD administration are generally unknown and strongly debated, as clearance from both tissue and body is an important factor in their toxicity.

\section{Iron oxide nanoparticles}

Iron oxide nanoparticles are based on magnetite $\left(\mathrm{Fe}_{3} \mathrm{O}_{4}\right)$, which has received the most attention for biomedical applications, or maghemite $\left(\gamma \mathrm{Fe}_{2} \mathrm{O}_{3}\right)$ molecules encapsulated in polysaccharide, synthetic polymers or monomer coatings [89]. These particles can be organized according to their hydrodynamic diameter into several categories: standard superparamagnetic iron oxide particles (SPIOs; 50-180 nm), very small superparamagnetic iron oxide particles $(10-50 \mathrm{~nm})$ and ultrasmall superparamagnetic iron oxide particles (USPIOs; $<10$ $\mathrm{nm})$. Nanoparticles $10-100 \mathrm{~nm}$ in diameter are too large to undergo renal elimination and as such have long blood half-lives. The utility of iron oxide nanoparticles is mainly due to their high biocompatibility and easy biodegradation in vivo, since after being metabolized, iron ions from these nanoparticles are added to the iron deposits or are incorporated by erythrocytes as part of the hemoglobin. Iron oxide nanoparticles below $30 \mathrm{~nm}$ in diameter are superparamagnetic and have been extensively explored for various diagnostic and potential therapeutic applications in the CNS. The ability of the USPIOs to cross the human BBB is significantly enhanced in the presence of an external magnetic force [90], and combining an external magnetic force with the disruption of tight junctions using D-mannitol significantly improves the permeability of negatively charged iron oxide nanoparticles through the BBB [91]. In addition, as has been described with other nanoparticles, the binding affinity of SPIOs can be modified by binding different molecules to their structure to produce nanoparticles with increased BBB crossing ability, targeting abilities (ligand-receptor mediated or other biomarkers) and fluorescent properties for optical imaging. Selective targeting of SPIO nanoparticles has been achieved by combining a SPIO with human recombinant EGF, leading to high intracellular incorporation in C6 glioblastoma cells [92]. SPION conjugated with cetuximab allowed binding and internalization into glioblastoma cells [93]. Moreover, lactoferrin-tethered magnetic double emulsion nanocapsules assembled with SPIOs and combined with cucurmin and doxorubicin efficiently suppress xenografted glioma cell tumor growth in brain tumor-bearing mice [94].

\section{Imaging \& diagnosis}

Among the magnetic particles, iron oxide nanoparticles have been the most investigated for CNS imaging. Specifically, SPIO nanoparticles, which provide negative contrast in a standard MRI T2 imaging sequence, are useful as MRI contrast agents to evaluate: areas of BBB dysfunction related to tumors and other neuroinflammatory pathologies; the cerebral vessels, using perfusion-weighted MRI sequences; and in vivo cellular tracking in CNS disease or injury.

Iron oxide nanoparticles have been used for diagnostic purposes with various diseases. Following intravenous injection, USPIOs are incorporated into macrophages via endocytosis [95]. The uptake of SPIO by phagocytic monocytes and macrophages provides a valuable in vivo tool by which MRI can be used to monitor the involvement of macrophages in inflammatory processes, such as multiple sclerosis, strokes or brain tumors. The macrophage SPIO-loaded MRI detection of tumor morphology might facilitate the surgical resection or biopsy of brain tumors. Moreover, the use of vectorization with antibodies of SPIO nanoparticles 
Table 1. Main characteristic of some nanoparticles utilized in vivo.

\begin{tabular}{|c|c|c|c|c|c|}
\hline & Member & Size $(n m)$ & $\begin{array}{l}\text { Application } \\
\text { in vivo }\end{array}$ & $\begin{array}{l}\text { Administration } \\
\text { pathway }\end{array}$ & Toxicity \\
\hline Natural polymers & Chitosan & $\approx 15$ & Antigen-carrier & Intravenous & Nontoxic, biodegradable \\
\hline \multirow[t]{2}{*}{$\begin{array}{l}\text { Lipids and cationic } \\
\text { lipids }\end{array}$} & $\begin{array}{l}\text { Liposomes decorated with } \\
\text { PEG }\end{array}$ & $\approx 100$ & Drug delivery & Intravenous & Nontoxic, biodegradable \\
\hline & Solid lipid nanoparticles & $40-200$ & Drug delivery & Intravenous & $\begin{array}{l}\text { Low toxicity, PMNs } \\
\text { activation }\end{array}$ \\
\hline \multirow[t]{2}{*}{ Synthetic polymers } & PEI nanogel & 50 & Drug delivery & Intraperitoneal & Low toxicity \\
\hline & PLGA & $\approx 150$ & Drug delivery & Intratympanic & Nontoxic, biodegradable \\
\hline \multirow[t]{2}{*}{$\begin{array}{l}\text { Carbon-based } \\
\text { nanoparticles }\end{array}$} & SWNT & $2-10$ & Drug delivery & Gastrogavage & $\begin{array}{l}\text { Oxidative stress } \\
\text { mitochondrial and } \\
\text { lysosomal damage }\end{array}$ \\
\hline & $\begin{array}{l}\text { PEGylated-MWNT } \\
\text { decorated with angiopep-2 }\end{array}$ & $10-120$ & Drug delivery & Intravenous & Nontoxic \\
\hline Quantum dots & $\mathrm{CdSe} / \mathrm{ZnS}$ & $2-10$ & Imaging & Stereotaxic & Oxidative stress \\
\hline $\begin{array}{l}\text { Iron oxide } \\
\text { nanoparticles }\end{array}$ & USPIOs & $<10$ & Imaging & Intravenous & Nontoxic \\
\hline
\end{tabular}

as cellular magnetic resonance contrast agents has been investigated to image lymphocyte populations within the CNS [96]. Two SPIO agents are clinically approved, namely ferumoxide and ferucarbotran [97]. Both ferumoxide and ferucarbotran are approved specifically for liver MRI, and clinical CNS imaging studies in humans have been also performed with USPIOs [98].

\section{Gold nanoparticles}

Gold nanoparticles (AuNPs) have been successfully used for drug and genetic material delivery in different tissues outside the CNS, but not in the CNS due to their limited distribution within the CNS following intravenous administration. Several strategies have been used to improve the CNS crossing abilities of AuNPs, such as an MRI-guided focused ultrasound technique that enhances the BBB permeability and brain delivery of AuNPs [99]. However, most of them explore a strategy based on incorporating several targeting peptides into the AuNPs, including: the peptide THRPPMWSPVWP, which binds to the transferrin receptor and increases the permeability of AuNPs in brain [100]; the incorporation of the transactivator of transcription (TAT) peptide to AUNPs to increase BBB crossing and deliver doxorubicin and gadolinium to brain tumor tissues [101]; the combination of the OX26 antibody with pegylated cationic solid lipid AuNPs, leading to the increased delivery of AUNPs to the brain [102] and by Angiopep-2 functionalization of pegylated AuNPs loaded with doxorubicin, which allowed in vivo distribution of nanoparticles into glioma brain xenografts and prolonged mice survival rate [103].

In addition, gold nanoparticles can also be used in cancer treatment. A new therapeutic approach involving the combination of radiation and gold nanoparticles has been tested to treat glioma, since AuNPs of the appropriate size can be used to enhance the effects of radiation in vivo [104,105]. Similarly, intravenous administration of AuNPs covalently linked to miR-182-based spherical nucleic acids (182-SNAs) penetrated the BBB and allowed 182-SNAs delivery in glioma brain xenografts, causing a reduction in tumor burden [106].

\section{Conclusion \& future perspective}

Given the aging population and increasing prevalence of neurological disorders, the demand for improved CNS therapeutics and diagnosis has increased considerably. The implementation of nanotechnology has revolutionized molecular neuroscience, leading to rapid advances in brain-specific drug and genetic 
material delivery, and in brain imaging and diagnosis. A great variety of nanoparticles have been designed and investigated for specific applications in the CNS. Table 1 shows the size, toxicity, administration, pathway and main potential biological application of the reviewed nanoparticles. High specific and multifunctional nanomaterials have contributed to the delivery of therapeutic, imaging and diagnostic molecules to the brain across the BBB, enabling substantial progress in the treatment, diagnosis and knowledge of CNS disorders. However, considering the complexity of the brain and the safety issues of nanomaterials, further research is needed so that those nanoparticle-mediated technologies that have shown such promise in vitro and in animal models can be developed for clinical applications. Targeted delivery is likely the main research direction of CNS nanomedicine. The availability of more specific and efficient targets is bound to facilitate the development of safer and more effective CNS-targeting nanomedicines.

\section{Open access}

This work is licensed under the Creative Commons AttributionNonCommercial 4.0 Unported License. To view a copy of this license, visit http://creativecommons.org/licenses/by-nc-nd/4.0/

\section{Financial \& competing interests disclosure}

This work has been supported, in part, by grants from MINECO, Spain (projects no. BFU2011-30161-C02-01 and BFU201459009-P) and CYTED (214RT0482). The authors have no other relevant affiliations or financial involvement with any organization or entity with a financial interest in or financial conflict with the subject matter or materials discussed in the manuscript apart from those disclosed.

No writing assistance was utilized in the production of this manuscript.

Executive summary

\section{Blood-brain barrier}

- It is a barrier that prevents the free passage of molecules with a molecular weight larger than 400-500 Da.

- Transport across this barrier can be facilitated by incorporating ligands for endogenous blood-brain barrier (BBB) receptors into the nanoparticles.

Natural polymers

- They can encapsulate hydrophobic compounds in its interior that are then protected from degradation.

- They comprise different types based in different building blocks including polysaccharides, collagen and cholesterol.

Lipids

- Lipidic nanoparticles are the most studied and clinically recognized drug nanocarriers.

- The potential of liposomes for CNS delivery is related to the ease of surface modification for targeted delivery.

- After intravenous administration there is vector aggregation leading to vector disintegration, genetic material release and degradation. This, together with the low efficiency of expression compared with viral vectors, has led to surface modifications to increase transfection efficiency and BBB crossing.

Polymeric nanoparticles

- It is an organic polymer with a high cationic-charge density potential due to the presence of multiple amino groups within its backbone that, in the absence of surface modification with target molecules, have a limited capacity to cross the BBB.

- Acrylic polymers and polyester-based nanoparticles offer the advantage of being rapidly degraded in vivo, which could reduce the toxicity caused by polymer accumulation in the CNS.

\section{Dendrimers}

- Dendrimers are repeatedly branched polymer molecules containing a cascade of branches grown from one or several cores. They contain three architectural domains: the core, to which the branches are attached; the shell of the branches surrounding the core and the multivalent surface formed by the branches' termini.

- Dendrimers and their cargo are endocytosed via a clathrin- and caveolae-mediated cellular internalization. Once inside the cell, dendrimers act as a proton sponge and escape from endosomes and lysosomes, releasing their cargo to the cytosol.

- Improving the BBB permeability of dendrimers has been addressed by coupling specific BBB-targeting ligands onto the surface of dendrimerssuch as transferrin, epidermal growth factor, rabies virus glycoprotein and angiopep-2 peptides.

\section{Carbon-based nanoparticles}

- Carbon nanoparticles are graphite sheets rolled into single or multiwalled tubes. They comprise fullerenes, carbon nanotubes and carbon nanohorns.

- To be used in biomedical applications, the nanotube's surface needs to be functionalized by incorporating different pendant units that increase its solubility.

- Toxicity of carbon-based nanoparticles remains an issue in biomedical applications since these materials can induce lipid peroxidation and oxidative stress as well as pulmonary toxicity. 
Executive summary (cont.)

\section{Quantum dots}

- Quantum dots are semiconductor nanoscale crystalline structures formed by colloidal sheets, composed of a metalloid crystalline core and a shell that shields the core.

- They have been used for drug and gene delivery to the CNS based on their ability to cross the BBB. However, quantum dots have emerged as a revolutionary imaging technology and have found diverse biomedical applications, including some in the CNS.

Iron oxide nanoparticles

- Iron oxide nanoparticles are based on magnetite or maghemite molecules encapsulated in polysaccharide, synthetic polymers or monomer coatings.

- Iron oxide nanoparticles have high biocompatibility and are easily biodegradable in vivo, since iron ions from these nanoparticles are added to the iron deposits or are incorporated by erythrocytes as part of the hemoglobin.

\section{Gold nanoparticles}

- Gold nanoparticles have a limited distribution within the CNS following intravenous administration.

- To improve the CNS crossing abilities of gold nanoparticles several targeting peptides have been incorporated into the nanoparticles.

\section{Conclusion}

- The implementation of nanotechnology has revolutionized molecular neuroscience, leading to rapid advances in brain-specific drug and genetic material delivery and in brain imaging and diagnosis.

- Targeted delivery is likely the main research direction of CNS nanomedicine. The availability of more specific and efficient targets is bound to facilitate the development of safer and more effective CNS-targeting nanomedicines.

\section{References}

Papers of special note have been highlighted as: $\bullet$ of interest; •• of considerable interest.

1 Hawkins MJ, Soon-Shiong P, Desai N. Protein nanoparticles as drug carriers in clinical medicine. Adv. Drug Deliv. Rev. 60(8), 876-885 (2008).

2 Lohcharoenkal W, Wang L, Chen YC, Rojanasakul Y. Protein nanoparticles as drug delivery carriers for cancer therapy. Biomed. Res. Int. 2014, 180549 (2014).

3 Posadas I, Guerra FJ, Ceña V. Nonviral vectors for the delivery of small interfering RNAs to the CNS. Nanomed. (Lond.) 5(8), 1219-1236 (2010).

4 Simko M, Mattsson MO. Interactions between nanosized materials and the brain. Curr. Med. Chem. 21(37), 4200-4214 (2014).

5 Doolittle ND, Muldoon LL, Culp AY, Neuwelt EA. Delivery of chemotherapeutics across the blood-brain barrier: challenges and advances. Adv. Pharmacol. 71, 203-243 (2014).

6 Xu G, Mahajan S, Roy I, Yong KT. Theranostic quantum dots for crossing blood-brain barrier in vitro and providing therapy of HIV-associated encephalopathy. Front. Pharmacol. 4, 140 (2013).

7 Abbott NJ, Ronnback L, Hansson E. Astrocyte-endothelial interactions at the blood-brain barrier. Nat. Rev. Neurosci. 7(1), 41-53 (2006).

8 Shilo M, Motiei M, Hana P, Popovtzer R. Transport of nanoparticles through the blood-brain barrier for imaging and therapeutic applications. Nanoscale 6(4), 2146-2152 (2014).

9 Demeule M, Currie JC, Bertrand Y et al. Involvement of the low-density lipoprotein receptor-related protein in the transcytosis of the brain delivery vector angiopep-2. J. Neurochem. 106(4), 1534-1544 (2008).

10 Malatesta M, Giagnacovo M, Costanzo M et al. Diaminobenzidine photoconversion is a suitable tool for tracking the intracellular location of fluorescently labelled nanoparticles at transmission electron microscopy. Eur. J. Histochem. 56(2), e20 (2012).

11 Peng YS, Lai PL, Peng $S$ et al. Glial cell line-derived neurotrophic factor gene delivery via a polyethylene imine grafted chitosan carrier. Int. J. Nanomed. 9, 3163-3174 (2014).

12 Skop NB, Calderon F, Levison SW, Gandhi CD, Cho CH. Heparin crosslinked chitosan microspheres for the delivery of neural stem cells and growth factors for central nervous system repair. Acta Biomater. 9(6), 6834-6843 (2013).

13 Nagpal K, Singh SK, Mishra DN. Nanoparticle mediated brain targeted delivery of gallic acid: in vivo behavioral and biochemical studies for protection against scopolamineinduced amnesia. Drug Deliv. 20(3-4), 112-119 (2013).

14 Ishii T, Asai T, Oyama D et al. Treatment of cerebral ischemia-reperfusion injury with PEGylated liposomes encapsulating FK506. FASEB J. 27(4), 1362-1370 (2012).

- $\quad$ PEG-modified liposomes have a larger circulation time and are more efficient.

15 Immordino ML, Dosio F, Cattel L. Stealth liposomes: review of the basic science, rationale, and clinical applications, existing and potential. Int. J. Nanomed. 1(3), 297-315 (2006).

16 Nel A, Xia T, Madler L, Li N. Toxic potential of materials at the nanolevel. Science 311(5761), 622-627 (2006).

17 Wong HL, Chattopadhyay N, Wu XY, Bendayan R. Nanotechnology applications for improved delivery of 
antiretroviral drugs to the brain. Adv. Drug Deliver. Rev. 62(4-5), 503-517 (2010).

18 de Boer AG, van der Sandt IC, Gaillard PJ. The role of drug transporters at the blood-brain barrier. Annu. Rev. Pharmacol. Toxicol. 43, 629-656 (2003).

19 Yusuf M, Khan M, Khan RA, Ahmed B. Preparation, characterization, in vivo and biochemical evaluation of brain targeted Piperine solid lipid nanoparticles in an experimentally induced Alzheimer's disease model. J. Drug Target. 21(3), 300-311 (2012).

20 Gaur PK, Mishra S, Bajpai M, Mishra A. Enhanced oral bioavailability of efavirenz by solid lipid nanoparticles: in vitro drug release and pharmacokinetics studies. Biomed. Res. Int. 2014, 363404 (2014).

21 Kuo YC, Wang CC. Cationic solid lipid nanoparticles with cholesterol-mediated surface layer for transporting saquinavir to the brain. Biotechnol. Prog. 30(1), 198-206 (2014).

22 Sahay G, Querbes W, Alabi C et al. Efficiency of siRNA delivery by lipid nanoparticles is limited by endocytic recycling. Nat. Biotechnol. 31(7), 653-658 (2013).

23 Frank RT, Aboody KS, Najbauer J. Strategies for enhancing antibody delivery to the brain. Biochim. Biophys. Acta 1816(2), 191-198 (2011).

24 Boado RJ, Pardridge WM. The Trojan horse liposome technology for nonviral gene transfer across the blood-brain barrier. J. Drug Deliv. 2011, 296151 (2011).

25 Zabel MD. Lipopeptide delivery of siRNA to the central nervous system. Methods Mol. Biol. 948, 251-262 (2013).

26 Savarala S, Brailoiu E, Wunder SL, Ilies MA. Tuning the self-assembling of pyridinium cationic lipids for efficient gene delivery into neuronal cells. Biomacromolecules 14(8), 2750-2764 (2013).

27 Zhong D, Jiao Y, Zhang Y et al. Effects of the gene carrier polyethyleneimines on structure and function of blood components. Biomaterials 34(1), 294-305 (2013).

28 Lee $\mathrm{CH}$, Kasala D, Na Y et al. Enhanced therapeutic efficacy of an adenovirus-PEI-bile-acid complex in tumors with low coxsackie and adenovirus receptor expression. Biomaterials 35(21), 5505-5516 (2014).

29 Zhang X, Oulad-Abdelghani M, Zelkin AN et al. Poly(Llysine) nanostructured particles for gene delivery and hormone stimulation. Biomaterials 31(7), 1699-1706 (2010).

30 Vauthier C, Dubernet C, Chauvierre C, Brigger I, Couvreur P. Drug delivery to resistant tumors: the potential of poly(alkyl cyanoacrylate) nanoparticles. J. Control. Release 93(2), 151-160 (2003).

31 Ulery BD, Nair LS, Laurencin CT. Biomedical applications of biodegradable polymers. J. Polym. Sci. B Polym. Phys. 49(12), 832-864 (2011).

32 Ogris M, Brunner S, Schuller S, Kircheis R, Wagner E. PEGylated DNA/transferrin-PEI complexes: reduced interaction with blood components, extended circulation in blood and potential for systemic gene delivery. Gene Ther. 6(4), 595-605 (1999).

33 Nguyen HK, Lemieux P, Vinogradov SV et al. Evaluation of polyether-polyethyleneimine graft copolymers as gene transfer agents. Gene Ther. 7(2), 126-138 (2000).
34 Tang GP, Zeng JM, Gao SJ et al. Polyethylene glycol modified polyethylenimine for improved CNS gene transfer: effects of PEGylation extent. Biomaterials 24(13), 2351-2362 (2003).

35 Kolter M, Ott M, Hauer C, Reimold I, Fricker G. Nanotoxicity of poly(n-butylcyano-acrylate) nanoparticles at the blood-brain barrier, in human whole blood and in vivo.J. Control. Release 197, 165-179 (2015).

36 Frank RT, Aboody KS, Najbauer J. Strategies for enhancing antibody delivery to the brain. Biochim. Biophys. Acta 1816(2), 191-198 (2011).

37 Solev IN, Balabanyan VY, Volchek IA et al. Involvement of BDNF and NGF in the mechanism of neuroprotective effect of human recombinant erythropoietin nanoforms. Bull. Exp. Biol. Med. 155(2), 242-244 (2013).

38 Jiang X, Xin H, Sha X et al. PEGylated poly(trimethylene carbonate) nanoparticles loaded with paclitaxel for the treatment of advanced glioma: in vitro and in vivo evaluation. Int. J. Pharm. 420(2), 385-394 (2011).

39 Klyachko NL, Manickam DS, Brynskikh AM et al. Crosslinked antioxidant nanozymes for improved delivery to CNS. Nanomedicine 8(1), 119-129 (2012).

40 Boylan NJ, Kim AJ, Suk JS et al. Enhancement of airway gene transfer by DNA nanoparticles using a $\mathrm{pH}$-responsive block copolymer of polyethylene glycol and poly-L-lysine. Biomaterials 33(7), 2361-2371 (2012).

41 Gerson T, Makarov E, Senanayake TH, Gorantla S, Poluektova LY, Vinogradov SV. Nano-NRTIs demonstrate low neurotoxicity and high antiviral activity against HIV infection in the brain. Nanomedicine 10(1), 177-185 (2014).

42 Ku B, Kim JE, Chung BH, Chung BG. Retinoic acidpolyethyleneimine complex nanoparticles for embryonic stem cell-derived neuronal differentiation. Langmuir 29(31), 9857-9862 (2013).

43 Fornaguera C, Dols-Perez A, Caldero G, Garcia-Celma MJ, Camarasa J, Solans C. PLGA nanoparticles prepared by nano-emulsion templating using low-energy methods as efficient nanocarriers for drug delivery across the blood-brain barrier. J. Control. Release 211, 134-143 (2015).

44 Jain A, Jain A, Garg NK et al. Surface engineered polymeric nanocarriers mediate the delivery of transferrin-methotrexate conjugates for an improved understanding of brain cancer. Acta Biomater. 24, 140-151 (2015).

45 Harrison J, Bartlett CA, Cowin G et al. In vivo imaging and biodistribution of multimodal polymeric nanoparticles delivered to the optic nerve. Small 8(10), 1579-1589 (2012).

46 Li J, Cai P, Shalviri A et al. A multifunctional polymeric nanotheranostic system delivers doxorubicin and imaging agents across the blood-brain barrier targeting brain metastases of breast cancer. ACS Nano 8(10), 9925-9940 (2014).

47 Tomalia DA, Baker H, Dewald J et al. A new class of polymers - starburst-dendritic macromolecules. Polym. J. 17(1), 117-132 (1985).

-• It is a classical reference on the early stages of dendrimer synthesis and applications. 
48 Albertazzi L, Gherardini L, Brondi M et al. In vivo distribution and toxicity of PAMAM dendrimers in the central nervous system depend on their surface chemistry. Mol. Pharm. 10(1), 249-260 (2013).

49 Tomalia DA, Christensen JA, Boas U. Dendrimers, dendrons and dendritic polymers. Cambridge University Press, UK, ISBN: 9760521515801 (2012).

50 Perez-Martinez FC, Guerra J, Posadas I, Ceña V. Barriers to non-viral vector-mediated gene delivery in the nervous system. Pharm. Res. 28(8), 1843-1858 (2011).

51 Shcharbin D, Janaszewska A, Klajnert-Maculewicz B et al. How to study dendrimers and dendriplexes III. Biodistribution, pharmacokinetics and toxicity in vivo. J. Control. Release 181, 40-52 (2014).

52 Lesniak WG, Mishra MK, Jyoti A et al. Biodistribution of fluorescently labeled PAMAM dendrimers in neonatal rabbits: effect of neuroinflammation. Mol. Pharm. 10(12), 4560-4571 (2013).

53 Mishra MK, Beaty CA, Lesniak WG et al. Dendrimer brain uptake and targeted therapy for brain injury in a large animal model of hypothermic circulatory arrest. ACS Nano 8(3), 2134-2147 (2014).

54 Gao X, Qian J, Zheng S et al. Up-regulating blood brain barrier permeability of nanoparticles via multivalent effect. Pharm. Res. 30 (10), 2538-2548 (2013).

55 Nyitrai G, Heja L, Jablonkai I, Pal I, Visy J, Kardos J. Polyamidoamine dendrimer impairs mitochondrial oxidation in brain tissue. J. Nanobiotechnol. 11, 9 (2013).

56 Wang S, Li Y, Fan J et al. The role of autophagy in the neurotoxicity of cationic PAMAM dendrimers. Biomaterials 35(26), 7588-7597 (2014).

57 Naha PC, Byrne HJ. Generation of intracellular reactive oxygen species and genotoxicity effect to exposure of nanosized polyamidoamine (PAMAM) dendrimers in PLHC-1 cells in vitro. Aquat. Toxicol. 132-133, 61-72 (2013).

58 Ciolkowski M, Petersen JF, Ficker M et al. Surface modification of PAMAM dendrimer improves its biocompatibility. Nanomedicine 8(6), 815-817 (2012).

59 Kannan S, Dai H, Navath RS et al. Dendrimer-based postnatal therapy for neuroinflammation and cerebral palsy in a rabbit model. Sci. Transl. Med. 4(130), 130ra46 (2012).

$60 \mathrm{Xu} \mathrm{L}$, Zhang H, Wu Y. Dendrimer advances for the central nervous system delivery of therapeutics. ACS Chem. Neurosci. 5(1), 2-13 (2014).

61 Perez-Carrion MD, Ceña V. Knocking down HMGB1 using dendrimer-delivered siRNA unveils its key role in NMDAinduced autophagy in rat cortical neurons. Pharm. Res. 30 (10), 2584-2595 (2013).

62 Patel SK, Gajbhiye V, Jain NK. Synthesis, characterization and brain targeting potential of paclitaxel loaded thiaminePPI nanoconjugates. J. Drug Target. 20 (10), 841-849 (2012).

$63 \mathrm{Ke}$ W, Shao K, Huang R et al. Gene delivery targeted to the brain using an Angiopep-conjugated polyethyleneglycolmodified polyamidoamine dendrimer. Biomaterials 30 (36), 6976-6985 (2009).
64 Neelov IM, Janaszewska A, Klajnert B et al. Molecular properties of lysine dendrimers and their interactions with Abeta-peptides and neuronal cells. Curr. Med. Chem. 20(1), 134-143 (2013).

65 Serramia MJ, Alvarez S, Fuentes-Paniagua E et al. In vivo delivery of siRNA to the brain by carbosilane dendrimer. J. Control. Release 200, 60-70 (2015).

66 Agrawal A, Min DH, Singh N et al. Functional delivery of siRNA in mice using dendriworms. ACS Nano 3(9), 2495-2504 (2009).

67 Kim ID, Shin JH, Kim SW et al. Intranasal Delivery of HMGB1 siRNA confers target gene knockdown and robust neuroprotection in the postischemic brain. Mol. Ther. 20(4), 829-839 (2012).

68 Albertazzi L, Brondi M, Pavan GM et al. Dendrimer-based fluorescent indicators: in vitro and in vivo applications. PLoS ONE 6(12), e28450 (2011).

69 Yan H, Wang J, Yi P et al. Imaging brain tumor by dendrimer-based optical/paramagnetic nanoprobe across the blood-brain barrier. Chem. Commun. (Camb.) 47(28), 8130-8132 (2011).

70 Lebedev AY, Cheprakov AV, Sakadzic S, Boas DA, Wilson DF, Vinogradov SA. Dendritic phosphorescent probes for oxygen imaging in biological systems. ACS Appl. Mater. Interfaces 1(6), 1292-1304 (2009).

71 Huang R, Han L, Li J et al. Chlorotoxin-modified macromolecular contrast agent for MRI tumor diagnosis. Biomaterials 32(22), 5177-5186 (2011).

72 Yan H, Wang L, Wang J et al. Two-order targeted brain tumor imaging by using an optical/paramagnetic nanoprobe across the blood brain barrier. ACS Nano 6(1), 410-420 (2012).

73 Matsumura S, Yuge R, Sato S et al. Ultrastructural localization of intravenously injected carbon nanohorns in tumor. Int. J. Nanomed. 9, 3499-3508 (2014).

74 Upadhyay RK. Drug delivery systems, CNS protection, and the blood brain barrier. Biomed. Res. Int. 2014, 869269 (2014).

75 Kostarelos K, Bianco A, Prato M. Promises, facts and challenges for carbon nanotubes in imaging and therapeutics. Nat. Nanotechnol. 4(10), 627-633 (2009).

76 Lacerda L, Bianco A, Prato M, Kostarelos K. Carbon nanotubes as nanomedicines: from toxicology to pharmacology. Adv. Drug Deliv. Rev. 58(14), 1460-1470 (2006).

-. Comprehensive toxicology and pharmacology review of carbon nanotubes.

77 Li N, Zhao Q, Shu C et al. Targeted killing of cancer cells in vivo and in vitro with IGF-IR antibody-directed carbon nanohorns based drug delivery. Int. J. Pharm. 478(2), 644-654 (2015).

78 Masserini M. Nanoparticles for brain drug delivery. ISRN Biochem. 2013, 238428 (2013).

79 Kafa H, Wang JT, Rubio N et al. The interaction of carbon nanotubes with an in vitro blood-brain barrier model and mouse brain in vivo. Biomaterials 53, 437-452 (2015). 
80 Nunes A, Bussy C, Gherardini L et al. In vivo degradation of functionalized carbon nanotubes after stereotactic administration in the brain cortex. Nanomed. (Lond.) 7(10), 1485-1494 (2012).

81 Al-Jamal KT, Gherardini L, Bardi G et al. Functional motor recovery from brain ischemic insult by carbon nanotubemediated siRNA silencing. Proc. Natl Acad. Sci. USA 108(27), 10952-10957 (2011).

82 Bardi G, Nunes A, Gherardini L et al. Functionalized carbon nanotubes in the brain: cellular internalization and neuroinflammatory responses. PLoS ONE 8(11), e80964 (2013).

83 Kostarelos K, Lacerda L, Pastorin G et al. Cellular uptake of functionalized carbon nanotubes is independent of functional group and cell type. Nat. Nanotechnol. 2(2), 108-113 (2007).

84 Dubertret B, Skourides P, Norris DJ, Noireaux V, Brivanlou $\mathrm{AH}$, Libchaber A. In vivo imaging of quantum dots encapsulated in phospholipid micelles. Science 298(5599), 1759-1762 (2002).

- One of the earliest descriptions of quantum dots imaging in vivo.

85 Law WC, Mahajan SD, Kopwitthaya A et al. Gene silencing of human neuronal cells for drug addiction therapy using anisotropic nanocrystals. Theranostics 2(7), 695-704 (2012).

Minami SS, Sun B, Popat K et al. Selective targeting of microglia by quantum dots. J. Neuroinflamm. 9, 22 (2012).

87 Boeneman K, Delehanty JB, Blanco-Canosa JB et al. Selecting improved peptidyl motifs for cytosolic delivery of disparate protein and nanoparticle materials. ACS Nano 7(5), 3778-3796 (2013).

Walters R, Kraig RP, Medintz I et al. Nanoparticle targeting to neurons in a rat hippocampal slice culture model. ASN Neuro 4(6), 383-392 (2012).

89 Laurent $\mathrm{S}$, Forge D, Port M et al. Magnetic iron oxide nanoparticles: synthesis, stabilization, vectorization, physicochemical characterizations, and biological applications. Chem. Rev. 108(6), 2064-2110 (2008).

Thomsen LB, Linemann T, Pondman KM et al. Uptake and transport of superparamagnetic iron oxide nanoparticles through human brain capillary endothelial cells. ACS Chem. Neurosci. 4(10), 1352-1360 (2013).

91 Sun Z, Worden M, Wroczynskyj Y et al. Magnetic field enhanced convective diffusion of iron oxide nanoparticles in an osmotically disrupted cell culture model of the bloodbrain barrier. Int. J. Nanomed. 9, 3013-3026 (2014).

92 Shevtsov MA, Nikolaev BP, Yakovleva LY et al. Superparamagnetic iron oxide nanoparticles conjugated with epidermal growth factor (SPION-EGF) for targeting brain tumors. Int. J. Nanomed. 9, 273-287 (2014).
93 Kaluzova M, Bouras A, Machaidze R, Hadjipanayis CG. Targeted therapy of glioblastoma stem-like cells and tumor non-stem cells using cetuximab-conjugated iron-oxide nanoparticles. Oncotarget 6(11), 8788-8806 (2015).

94 Fang JH, Lai YH, Chiu TL, Chen YY, Hu SH, Chen SY. Magnetic core-shell nanocapsules with dual-targeting capabilities and co-delivery of multiple drugs to treat brain gliomas. Adv. Healthc. Mater. 3(8), 1250-1260 (2014).

95 Wang QB, Han Y, Jiang TT et al. MR Imaging of activated hepatic stellate cells in liver injured by $\mathrm{CCl} 4$ of rats with integrin-targeted ultrasmall superparamagnetic iron oxide. Eur. Radiol. 21(5), 1016-1025 (2011).

96 Luchetti A, Milani D, Ruffini F et al. Monoclonal antibodies conjugated with superparamagnetic iron oxide particles allow magnetic resonance imaging detection of lymphocytes in the mouse brain. Mol. Imaging 11(2), 114-125 (2012).

97 Wang YX. Superparamagnetic iron oxide based MRI contrast agents: current status of clinical application. Quant. Imaging Med. Surg. 1(1), 35-40 (2011).

98 Saleh A, Schroeter M, Jonkmanns C, Hartung HP, Modder $\mathrm{U}$, Jander S. In vivo MRI of brain inflammation in human ischaemic stroke. Brain 127(Pt 7), 1670-1677 (2004).

99 Etame AB, Diaz RJ, O'Reilly MA et al. Enhanced delivery of gold nanoparticles with therapeutic potential into the brain using MRI-guided focused ultrasound. Nanomedicine 8(7), 1133-1142 (2012).

100 Dixit S, Novak T, Miller K, Zhu Y, Kenney ME, Broome AM. Transferrin receptor-targeted theranostic gold nanoparticles for photosensitizer delivery in brain tumors. Nanoscale 7(5), 1782-1790 (2015).

101 Cheng Y, Dai Q, Morshed RA et al. Blood-brain barrier permeable gold nanoparticles: an efficient delivery platform for enhanced malignant glioma therapy and imaging. Small 10(24), 5137-5150 (2014).

102 Liu Y, He M, Niu M et al. Delivery of vincristine sulfateconjugated gold nanoparticles using liposomes: a lightresponsive nanocarrier with enhanced antitumor efficiency. Int. J. Nanomed. 10, 3081-3095 (2015).

103 Ruan S, Yuan M, Zhang L et al. Tumor microenvironment sensitive doxorubicin delivery and release to glioma using angiopep-2 decorated gold nanoparticles. Biomaterials 37 , 425-435 (2015).

104 Bobyk L, Edouard M, Deman P et al. Photoactivation of gold nanoparticles for glioma treatment. Nanomedicine 9(7), 1089-1097 (2013).

105 Antosh MP, Wijesinghe DD, Shrestha S et al. Enhancement of radiation effect on cancer cells by gold-pHLIP. Proc. Natl Acad. Sci. USA 112(17), 5372-5376 (2015).

106 Kouri FM, Hurley LA, Daniel WL et al. miR-182 integrates apoptosis, growth, and differentiation programs in glioblastoma. Genes Dev. 29(7), 732-745 (2015). 